Nota clínica

\title{
Síndrome del cascanueces posterior: Vena renal retroaórtica asociada a fístula arteriovenosa y carcinoma renal. A propósito de un caso y revisión de la literatura
}

\author{
Carlos Muller Arteaga, Sergio Martín Martín, José R. Cortiñas González, José A. González Fajardo*, \\ Ernesto Fernández del Busto
}

Servicio de Urología. Servicio de Angiología y *Cirugía Vascular. Hospital Clínico Universitario de Valladolid.

\begin{abstract}
Resumen
Las alteraciones vasculares del retroperitoneo son poco frecuentes y sin embargo, cada vez más diagnosticadas. Presentamos un caso de asociación muy poco frecuente en el que se relaciona una malformación congénita vascular con la presencia de una fístula arteriovenosa renal y un carcinoma renal izquierdo en una paciente que sólo presentó hematuria. Estas variaciones vasculares deben ser consideradas en el momento de planificar una cirugía con la finalidad de hacerla más segura y con menos complicaciones. Se discute el caso y se revisa la literatura.
\end{abstract}

Palabras clave: Cáncer de riñón. Fístula urovascular. Vena renal retroaórtica. Síndrome del cascanueces.

Posterior nutcracker syndrome: retroaortic renal vein associated with arteriovenous fistula and renal cancinoma. Report of a case and review of literature

\begin{abstract}
Vascular retroperitoneal alterations are little frequent and nevertheless, more diagnosed. We presented a case of association very little frequents in which a vascular congenital malformation is related to the presence of a renal arteriovenous fistula and a left renal carcinoma in a patient who only presented hematuria. These vascular variations must be considered at the moment for planning a surgery with the purpose of making it safer and to avoid complications. The case is discussed and literature is reviewed.
\end{abstract}

Keywords: Renal cancer. Urovascular fistula. Retroaortic renal vein. Nutcracker syndrome.

$\mathrm{L}$ as anomalías vasculares del retroperitoneo son poco frecuentes; sin embargo, cada vez son más diagnosticadas por el uso de diferentes métodos de diagnóstico por imágenes. El urólogo debe conocer las posibles variaciones a nivel vascular con la finalidad de evitar complicaciones y realizar una cirugía más segura.

Presentamos un caso de asociación muy poco frecuente en el que una malformación anatómica se relaciona con una fístula arteriovenosa en el contexto de un carcinoma renal.

Se discute el caso y se revisa la literatura.

\section{CASO CLINICO}

Se trata de una paciente de 71 años de edad quien ingresa procedente del Servicio de Urgencias por presentar hematuria persistente. En el examen físico no se revelan hallazgos significati- vos, no soplos abdominales. Los resultados de los análisis de sangre y coagulación son normales y en el sistemático de orina se detecta la presencia de hematuria. Se realizan estudios complementarios:

a) Urografías (UIV) en las que se observa una deformidad del cáliz superior del riñón izquierdo con efecto masa sobre el mismo y el cáliz medio e irregularidades en la pelvis renal izquierda sin defectos de repleción que pudieran estar en relación con múltiples improntas vasculares (Fig. 1).

b) Tomografía computarizada (TC) en la que se evidencia una masa en el polo superior del riñón izquierdo asociada a una probable fístula arteriovenosa (AV) y que capta contraste sugestiva de hipernefroma (Fig. 2).

Además se evidencia la presencia de una vena renal izquierda (VRI) retroaórtica (Fig. 3). 


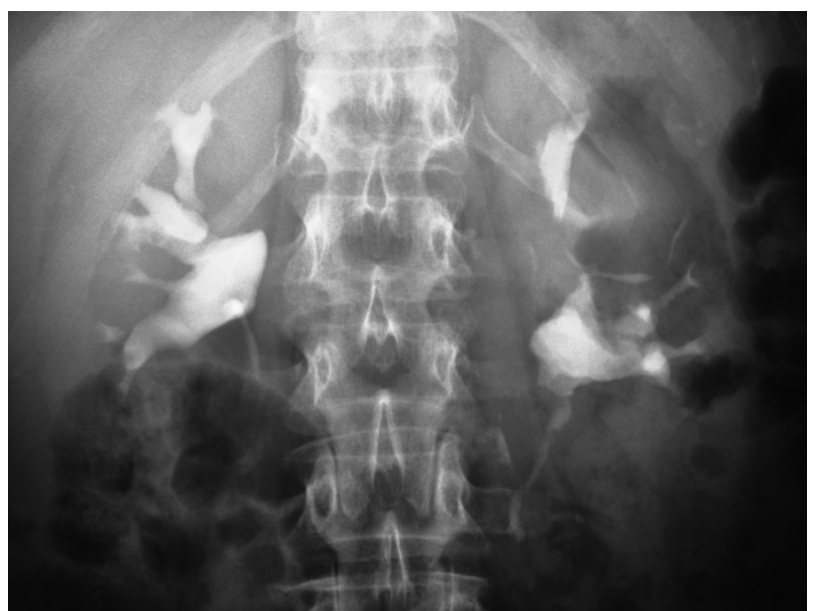

FIGURA 1. Urografia en la que se observa deformidad del cáliz superior del riñón izquierdo com efecto masa.

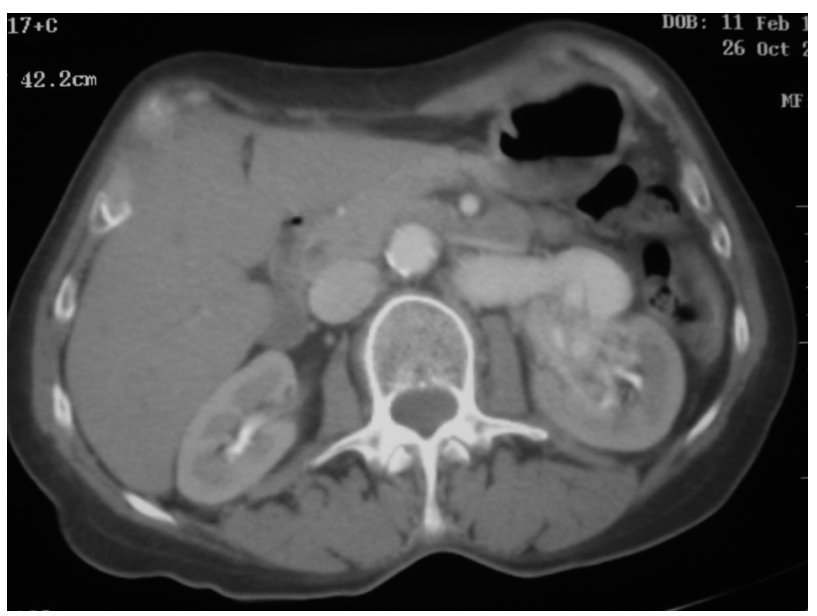

FIGURA 2. TAC: Se observa masa en el polo superior del riñón izquierdo asociada a una probable fistula arteriovenosa sugestiva de hipernefroma.

c) Arteriografía (Fig. 4) en la que se evidencia una fístula arteriovenosa de alto flujo en el tercio medio de dicho riñón de aproximadamente $5 \mathrm{~cm}$ con aneurisma venoso asociado y una VRI retroaórtica de gran calibre $(4 \mathrm{~cm})$; también llama la atención la presencia de una zona hipervascularizada que rodea la malformación $\mathrm{AV}$ con neoformación de vasos $\mathrm{y}$ arterias capsulares hipertrofiadas sugestivas de una neoplasia renal asociada.

La paciente es intervenida quirúrgicamente realizándose nefrectomía radical izquierda con abordaje transperitoneal. La evolución posterior es satisfactoria siendo dada de alta sin complicaciones postoperatorias a los 7 días.

El resultado de Anatomía patológica describe una lesión sólida con áreas de hemorragia de 5 x 4

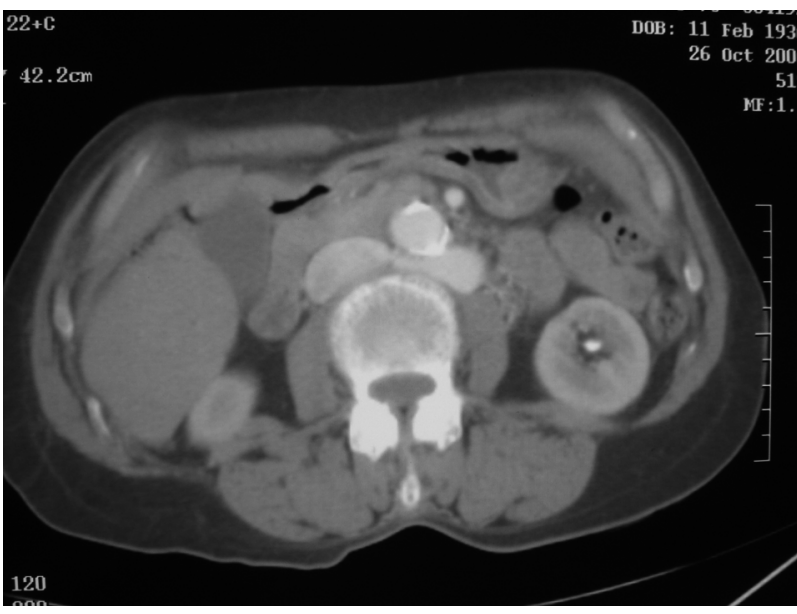

FIGURA 3. TAC: Vena renal izquierda retroaórtica.

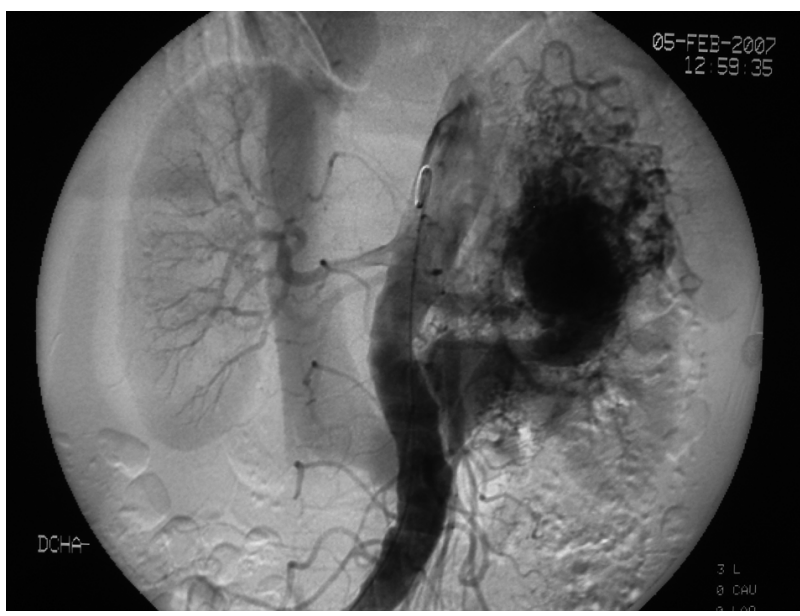

FIGURA 4. Arteriografia: fistula arteriovenosa con aneuris ma venoso asociado y VRI retroaórtica, además zona hipervascularizada sugestiva de una neoplasia renal asociada.

cm. en polo superior que afecta corteza y médula sin afectar cápsula renal, relacionada con vasos dilatados, tortuosos y congestivos, concluye: Carcinoma renal de células claras y malformación AV (T1b,NO, MO: Estadio I).

\section{DISCUSIÓN}

Las alteraciones del sistema venoso en el retroperitoneo pueden ser tanto congénitas como adquiridas y por este motivo se debe conocer la embriología y las diferentes patologías que pueden afectar la anatomía de este sistema, con la finalidad de evitar complicaciones durante la cirugía ${ }^{1,2}$.

La mayor parte de las anomalías venosas reciben estudio y tratamiento por parte de los cirujanos vasculares a través de las intervenciones aortoilíacas; 
sin embargo, el urólogo esta obligado a conocer las variaciones ya que debe enfrentarse a ellas en el transcurso de la cirugía retroperitoneal y, porque en muchos casos es consultado como parte de un equipo multidisciplinario en la resolución de diferentes patologías del retroperitoneo ${ }^{1}$.

Las descripciones de anomalias de la Vena Cava Inferior (VCI) se remontan a 1793 cuando Abernethy describió la presencia de un shunt mesocava y la continuación de la VCI en la ázigos en un lactante de 10 meses $^{3}$.

La embriología de la VCI y de las venas renales forma parte de un complejo proceso de formación, regresiones parciales y anastomosis vasculares de los pares de las 3 venas primitivas fetales (derecha, media e izquierda) ${ }^{1,3}$ que sucede entre la sexta y la décima semana de gestación ${ }^{2,3}$. De esta forma, se entiende como pequeñas modificaciones pueden dar origen a grandes cambios anatómicos en el desarrollo posterior de todo el sistema de drenaje venoso del retroperitoneo. Además, se explica también la existencia de anomalías vasculares asociadas ${ }^{2}$.

La alteración congénita más común de la VCI es la presencia de múltiples venas renales, generalmente en número de 2 , y que varía desde 4.22 a $26 \%$ en las afluentes derechas y, de 0 a $2,6 \%$ en las venas del lado izquierdo, con una incidencia global de aproximadamente un 5\%. También están descritas: la trasposición de VCI (0-0,5\%), su duplicación (0,02-3\%), la VRI circunaórtica $(0,088-8,7 \%)$, la interrupción de la VCI por la ázigos o hemiázigos $(0,1-4,3 \%)$ y, la VRI retroaórtica (como en el caso que nos ocupa) $(0,64-3,7 \%)^{1,3,4}$. Las anomalías adquiridas son generalmente derivadas de las alteraciones producidas por los shunts en los casos de hipertensión portal ${ }^{1}$.

El Síndrome del cascanueces (SC) fue descrito inicialmente por De Schepper en 1972 que lo denominó "Síndrome de atrapamiento de la VRI"5,6, por este motivo algunos autores prefieren denominar a esta patología como Fenómeno del cascanueces, englobando bajo este concepto a la "compresión de la VRI por cualquier estructura anatómica adyacente"4.

Posteriormente, algunos autores han clasificado este síndrome en: SC anterior (compresión de la VRI entre la aorta y la arteria mesentérica superior) y, posterior o también llamado pseudosíndrome del cascanueces (compresión de la VRI entre la Aorta y los cuerpos vertebrales) ${ }^{4,5,7}$. Se ha descrito que el $\mathrm{SC}$ anterior se produce en la mayoría de los casos por una ptosis renal izquierda y/o la presencia de una Arteria mesentérica superior con un nacimiento anómalo; mientras que el SC posterior se debe a la persistencia de la rama posterior del collar periaórtico fetal que forman los vasos renales durante la gestación con degeneración de la rama anterior que normalmente es la que debería persistir ${ }^{4}$.

El SC se presenta como una patología generalmente asintomática ${ }^{5,6}$ ya que la VRI tiene una gran adaptabilidad a las alteraciones del flujo ${ }^{4}$. En algunos casos puede cursar con hematuria, dolor en flanco, y dilataciones vasculares en el sistema venoso afluente debido al aumento de presión para el drenaje (varicocele, varicosidades pelvianas, etc.) ${ }^{4-7}$; generalmente se presenta con similar distribución en ambos sexos ${ }^{5}$.

También han sido descritas fístulas AV intrarrenales asociadas al $\mathrm{SC}^{5}$, como en el caso que presentamos.

La presencia de una gran fístula en los vasos renales fue descrita por primera vez en 1928 por Varela. Las fístulas AV se clasifican en: congénitas y adquiridas, siendo más frecuentes estas últimas (70\%); y en cuanto a su etiología, la causa más común es la secundaria a intervenciones quirúrgicas sobre el riñón (38\%). Sólo un 16\% de las fístulas AV se asocian a carcinomas renales ${ }^{8}$.

Las alteraciones de la VCI suelen ser asintomáticas $^{3}$. Las manifestaciones clínicas más comunes descritas en la literatura en los casos de asociación entre fístula $\mathrm{AV}$ y carcinoma renal incluyen: soplos intra-abdominales, cardiomegalia e hipertensión diastólica ${ }^{8}$. La hematuria en las neoplasias renales se presenta en aproximadamente el 60\% de los casos, cuando se ha relacionado la presencia de tumores renales asociados a fístulas, como en el caso que describimos, este porcentaje es de un 15\% ( 2 pacientes de una revisión de 13 pacientes) ${ }^{8}$.

Las formas de diagnóstico preoperatorio de las anomalías de la VCI y de la vascularización renal son: la TC, la Resonancia Magnética nuclear, las reconstrucciones de cortes tomográficos en 3 dimensiones $^{1-7}$, la arteriografía ${ }^{8}$, ECO-doppler ${ }^{4-6}$, la flebografía de VCI con la medida de los gradientes de presión ${ }^{4-7}$, etc.

El tratamiento del SC posterior esta justificado sólo en presencia de sintomatología y es la trasposición de la VRI a una posición anterior ${ }^{7}$, sin embargo, en nuestro caso ante la presencia de la patología renal asociada el tratamiento realizado fue la nefrectomía radical. 
El uso de métodos de diagnóstico por imagen de una forma generalizada en el contexto de patologias retroperitoneales, sobre todo en los tumores de riñón, trae como consecuencia que cada vez se identifiquen una mayor cantidad de alteraciones de la vascularización renal y esto permite al urólogo planificar una cirugía más segura y con menos complicaciones.

\section{REFERENCIAS}

1. Matsuura T, Morimoto Y, Nose K, Hara Y, Akiyama T, Kurita T. Venous abnormalities incidentally accompanied by renal tumors. Urol Int. 2004:73(2):163-168.

2. Pascual Mateo C, Luján Galán M, Rodríguez García N, Gómez de Vicente JM, Santos Arrontes D, Aguilar E, et al. Vena cava renal izquierda en un paciente con carcinoma de células renales. Actas Urol. Esp. 2005;29(7):693-695.

3. Bass JE, Redwine MD, Kramer LA, Huynh PT, Harris JH Jr. Spectrum of Congenital Anomalies of the Inferior Vena Cava: Cross-sectional Imaging Findings. Radiographics. 2000;20(3): 639-652.

4. Cuéllar i Calàbria $\mathrm{H}$, Quiroga Gómez S, Sebastià Cerqueda C, Boyé de la Presa R, Miranda A, Alvarez-Castells A. Nutcracker or left renal vein compression phenomenon: multidetector computed tomography findings and clinical significance. Eur. Radiol. 2005(15):1745-1751.

5. Santos Arrontes D, Salgado Salinas R, Chiva Robles V, Gómez de Vicente JM, Fernández González I, Costa Subias J, et al. Síndrome del cascanueces. A propósito de un caso y revisión de la literatura. Actas Urol Esp. 2003;27(9):726-731.

6. Fu WJ, Hong BF, Gao JP, Xiao YY, Yang Y, Cai W, et al. Nutcracker phenomenon: A new diagnostic method of multislice computed tomography angiography. Int J Urol. 2006;13(7):870873.

7. Ali-El-Dein B, Osman Y, Shehab El-Din AB, El-Diasty T, Mansour O, Ghoneim MA. Anterior and posterior nutcracker syndrome: a report on 11 cases. Trasp Proc. 2003;35(2):851-853.

8. Holmes SA, Ball AJ. Arteriovenous fistula associated with adenocarcinoma of the kidney. Urol Int. 1991;47(2):81-83.

Correspondencia autor: Carlos Muller Arteaga

Servicio de Urología. Hospital Clínico Universitario de Valladolid. Av. Ramón y Cajal, s/n - 47003 Valladolid Tel.:983 420000 Ext. 20226

E-mail autor: urologo@hotmail.com Información artículo: Nota clínica

Trabajo recibido: abril 2007

Trabajo aceptado: mayo 2007 